\title{
La equidad, elemento esencial para la inclusión en educación
}

\author{
Equity, an essential element for inclusion in education
}

\section{Mayté Pérez Vences ${ }^{\mathrm{a}}$-Adoración Barrales Villegas ${ }^{\mathrm{b}}$-Regina Dajer Torres ${ }^{\mathrm{c}}$-Lilia Esther Guerrero Rodríguez ${ }^{\mathrm{d}}$}

a Observatorio del Observatorio Regional de Calidad y Equidad de la Educación Superior, Facultad de Pedagogía, Universidad Veracruzana, Poza Rica, México. Contacto: mayperez@uv.mx

${ }^{b}$ Responsable del Observatorio Regional de Calidad y Equidad de la Educación Superior, Facultad de Pedagogía, Universidad Veracruzana, Poza Rica, México. Contacto: abarrales@uv.mx

c Observatorio del Observatorio Regional de Calidad y Equidad de la Educación Superior, Facultad de Pedagogía, Universidad Veracruzana, Poza Rica, México. Contacto: rdajer@uv.mx

d Observatorio del Observatorio Regional de Calidad y Equidad de la Educación Superior, Facultad de Pedagogía, Universidad Veracruzana, Poza Rica, México. Contacto: liguerrero@uv.mx

Recibido: 21 de septiembre de 2020

Aceptado: 15 de octubre de 2020

RESUMEN: El Observatorio Regional de Calidad y Equidad de la Educación Superior (ORACLE), instalado en la Facultad de Pedagogía, región Poza Rica-Tuxpan, de la Universidad Veracruzana, a cargo del Cuerpo Académico Gestión e Investigación Educativa Transdisciplinaria, surge a invitación de la Universidad Autónoma de Barcelona bajo la financiación del programa ERASMUS+, su objetivo: crear observatorios que amplíen la gestión de la calidad y la equidad proyectándolas a todas las funciones y estamentos que operan en la institución. Para analizar la operatividad del principio de equidad revisamos: 1) la Ley Orgánica, 2) la Ley de Autonomía y 3) el Código de Ética. En este escrito mostraremos los resultados del diagnóstico realizado por el ORACLE, en las diferentes voces de sus actores, identificando áreas de oportunidad y, con ello, visibilizando la importancia de transversalizar 
la equidad como promotora de la calidad para garantizar procesos equitativos e igualitarios a la comunidad universitaria.

Palabras clave: Equidad; inclusión; vulnerabilidad; educación.

ABSTRACT: The Regional Observatory of Quality and Equity in Higher Education (ORACLE) installed on the Pedagogy Faculty of the University of Veracruz in Poza Rica - Tuxpan campus, in charge of the "Transdisciplinary Educational Management and Research" research group, which arises from an invitation from the Autonomous University of Barcelona under the ERASMUS+ program funding. Its main goal: to create observatories that expand the quality and equity management, projecting them to all functions and levels that operate in the institution. To analyze the operation of the equity principle, we reviewed: 1) the Organic Law, 2) the Autonomy Law and 3) the Code of Ethics. In this writing we will show the results of the diagnosis made by ORACLE, in the different voices of its actors, identifying opportunity areas and with it, making visible the importance of mainstreaming equity as a promoter of quality to guarantee equitable and egalitarian processes to the university community.

Keywords: Equity; Inclusion; Vulnerability, Education

\section{Introducción}

工 a inclusión es un concepto surgido en los años noventa y a nivel mundial se trabaja en diferentes rubros, incluyendo el ámbito educativo. Desafortunadamente a pesar de las diferentes acciones emprendidas todavía se siguen observando insuficiencias en la incorporación de sectores importantes de la sociedad, como son los grupos socialmente vulnerables derivados de la discapacidad, pobreza, etnicidad o género.

México no es ajeno a las pretensiones de alcanzar una inclusión educativa, por ello es prioritario entender que se trata de un proceso que busca garantizar el derecho a una educación de calidad para todos y, para lograrlo se requiere del esfuerzo de todos los componentes de la sociedad e integrar a aquellos que son marginados y segregados por diversas circunstancias. 
La UNESCO define la educación inclusiva

\begin{abstract}
como el proceso de identificar y responder a la diversidad de las necesidades de todos los estudiantes a través de la mayor participación en el aprendizaje, las culturas y las comunidades, y reduciendo la exclusión en la educación. Involucra cambios y modificaciones en contenidos, aproximaciones, estructuras y estrategias, con una visión común que incluye a todos los niños del rango de edad apropiado y la convicción de que es la responsabilidad del sistema regular, educar a todos los niños/as. (Rodríguez; 2019: 2o párrafo)
\end{abstract}

Por lo tanto, la educación inclusiva se fundamenta en el principio de que cada niño/a es único y diferente a los demás; tiene características, intereses, capacidades y necesidades de aprendizaje distintos por lo que es un deber de la educación, las instituciones y sistemas educativos atender a estas peculiaridades, así como especificidades de cada individuo.

Es claro que para que las instituciones educativas sean inclusivas se requiere de principios y valores encaminados a erradicar cualquier acontecimiento donde se atente contra la integridad humana, es por ello que la equidad se hace indispensable al interior de las instituciones educativas.

Ahora bien, realizar un análisis de la equidad como un elemento esencial para lograr una inclusión en la educación, es el objetivo principal de dar a conocer los resultados del diagnóstico realizado por ORACLE, en relación a como perciben la equidad los miembros de la comunidad universitaria; de entrada se presenta una revisión general de las políticas educativas institucionales que se han implementado para favorecer la equidad, así como mostrar los resultados y concluir en una serie de reflexiones que se derivan del análisis de los indicadores que conforman el diagnóstico.

\title{
Desarrollo
}

En México, los últimos años se ha hecho énfasis en la importancia de ser inclusivos en todos los ámbitos de la vida cotidiana, el caso de la educación no es la excepción, sobre todo porque se tiene la inquietud de estar en sintonía con las políticas internacionales que se han establecido con este fin.

En la primera parte, se hace una breve revisión de lo que se ha realizado en el plano institucional en la Universidad Veracruzana, en relación a las políticas que fomentan la equidad como elemento indispensable para lograr la inclusión.

En la segunda parte se muestran los resultados de un diagnóstico institucional realizado por el Observatorio ORACLE, con la intención de conocer el impacto de las políticas que la 
Observatorios - Observatorio Regional de Calidad y Equidad de la Educación Superior

Universidad Veracruzana ha implementado para fomentar la equidad en la vida cotidiana de la comunidad universitaria.

\section{Revisión de las políticas de equidad en la Universidad Veracruzana}

Existen intenciones que marcan gran preocupación en el tema de la educación, existen compromisos adquiridos que conllevan grandes esfuerzos y trabajos coordinados, en otras palabras, una alineación hacia las políticas internacionales, nacionales y estatales como una oportunidad para alcanzar avances positivos en la mejora de la calidad educativa.

En lo que respecta a la Universidad Veracruzana la política y la normativa institucional en torno a la equidad e inclusión educativas se encuentra fundamentada en los siguientes documentos.

\section{Ley Orgánica y Ley de Autonomía}

La Ley Orgánica, así como la Ley de Autonomía, no manejan específicamente los conceptos de equidad y/o inclusión, sin embargo, sientan las bases para que la Universidad pueda generar convenios, programas, proyectos y reglamentos relacionados con cualquier temática (incluida la equidad y la inclusión) con otras instituciones de nivel superior públicas y privadas, a través de sus funcionarios, en las figuras de su rectora y abogado general.

\section{Código de ética}

El Código de Ética de la Universidad Veracruzana es un documento que recupera los principios y valores que deben orientar la conducta de todos los miembros de la comunidad universitaria. Su estructura está compuesta por dos títulos: Normas generales y 10 capítulos.

A continuación, hacemos hincapié en los capítulos que dan sustento al presente trabajo, como son la equidad, la igualdad y la inclusión. En el Capítulo I: Dignidad, Igualdad y No Discriminación se define la dignidad de la siguiente manera:

En cuanto principio, la dignidad conlleva el reconocimiento de que todas las personas son valiosas en sí mismas y merecen respeto. Un respeto que no está condicionado a ninguna circunstancia y que implica que las personas no deben ser tratadas como objetos o como medios para la consecución de cualesquiera fines (Universidad Veracruzana, 2016, p. 7). 
En otras palabras, la dignidad es una cualidad con la que todos los seres humanos nacemos, es una capacidad, una característica que nos hace únicos y merecedores de respeto, la universidad tiene la misión de garantizar que todos sus miembros sean tratados con dignidad. En lo que se refiere a la igualdad y la no discriminación, el Código de Ética menciona que:

...la igualdad es un principio ético y jurídico reconocido universalmente que comprende la prohibición absoluta de discriminación de las personas, por acción u omisión, por cualquiera de los motivos prohibidos en la constitución federal o en los tratados internacionales de los que México sea parte o por cualquier otro que atente contra la dignidad humana (Universidad Veracruzana, 2016, p. 7-8)

Finalmente, en el Capítulo X del Código de Ética se menciona que:

...la equidad es un principio ético que busca la justicia en la igualdad, que pretende avanzar en la construcción de una sociedad o comunidad más justa, en la que la aplicación absoluta del principio de igualdad no se traduzca en injusticia al no tener en cuenta las diferencias existentes entre las personas y los grupos. (Universidad Veracruzana, 2016, p. 20)

Lo anterior implica un respeto mutuo a las diferencias y necesidades de los colectivos que integran la comunidad académica.

Derivado de la normativa, que se ha mencionado existen como estrategias algunos programas institucionales que desarrollan el principio de equidad en la dinámica de comunidad universitaria, entre ellas destacamos de manera enunciativa más no limitativa, al Programa de Trabajo Estratégico (PTE) 2017-2021: Pertenencia y Pertinencia; el Centro de Desarrollo Humano e Integral de los Universitarios (CENDHIU); Programa Universitario por una Universidad Incluyente e Integradora de Personas con Discapacidad y El Sistema Universitario de Gestión Integral del Riesgo (SUGIR).

El PTE 2017-2021 promueve la equidad educativa ofreciendo igualdad de oportunidades a los alumnos para realizar estudios en programas reconocidos por su calidad; así como la incorporación de los enfoques intercultural y de equidad de género, el respeto por las diferencias, los derechos humanos y la dimensión internacional en las funciones institucionales. (Universidad Veracruzana, 2018)

El CENDHIU aprobado por el Consejo Universitario en el año 2010, cuya finalidad es:

Promover una Universidad Inclusiva donde predominen los procesos de formación, aprendizaje y toma de conciencia, que permitan posicionar los valores de igualdad, no discriminación y respeto a los derechos humanos, así como visibilizar y disminuir las barreras de aprendizaje, 
académicas, sociales y profesionales, particularmente de estudiantes y miembros de la comunidad universitaria que viven con discapacidad sensorial, motriz e intelectual. (Universidad Veracruzana, 2019, párr. 1).

Otra de las estrategias es el Programa Universitario por una Universidad Incluyente e Integradora de Personas con Discapacidad tiene como propósito:

\begin{abstract}
...contribuir al desarrollo de una Educación Inclusiva que permita la plena integración e inclusión de las personas con discapacidad a la comunidad Universitaria, es también, el formar futuros profesionales con un perfil que les permita utilizar sus conocimientos, actitudes, y habilidades para la construcción de sociedades accesibles para todos. (Universidad Veracruzana, 2016, p.1).
\end{abstract}

Una estrategia más es el SUGIR que tiene el fin de: “establecer y desarrollar la capacidad institucional para responder y contribuir de manera permanentemente en la construcción de una cultura del autocuidado y del cuidado colectivo, desde una filosofía de gestión integral del riesgo congruente con la sustentabilidad". (Universidad Veracruzana, 2019, párr. 1).

Finalmente, como pudimos constatar la Universidad Veracruzana se ha preocupado por incorporar a su política y a su dinámica intitucional el desarrollo del principio de equidad, en la intención de proteger a todos y cada unos de los miembros de su comunidad académica.

\title{
Metodología
}

El observatorio se caracteriza por una visión amplia de la realidad; en nuestro caso buscamos, además, contribuir a resolver una situación a partir de un conjunto de acciones integradas para el logro de objetivos a determinado plazo.

Como observatorio nos insertamos en el quehacer educativo mediante la investigación acción siguiendo el modelo de Kemmis (1992), buscando la transformación y mejora de la gestión educativa; el hecho de fundamentarnos en este proceso se debe que al verla como una espiral de ciclos de investigación y acción (ver fig. 1) constituidos en fases como: planificar, actuar, observar y reflexionar, nos facilita una herramienta para su práctica interventora, con el fin de encontrar soluciones a las problemáticas que se presenten en la institución educativa y transformar su propia realidad. 


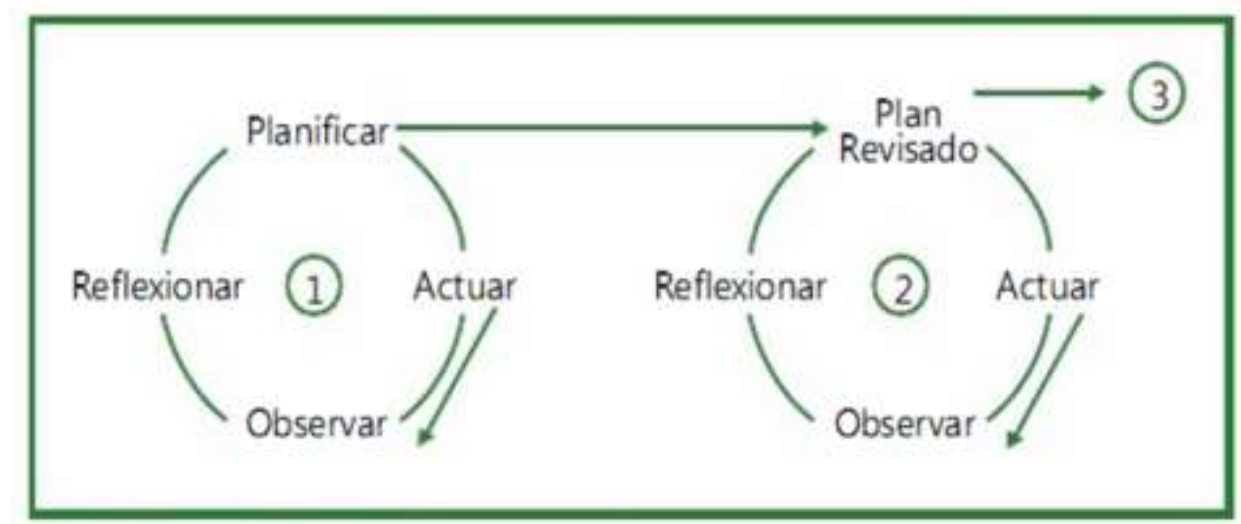

Figura 1. La espiral de ciclos de la investigación-acción. Fuente: Latorre (2005) p. (32) recuperado de http://ri.uaemex.mx/bitstream/handle/20.500.11799/57864

En la práctica estas fases las desarrollamos de la siguiente forma:

1. Planificación y revisión del contexto de actuación. En la actualidad una de las preocupaciones de las instituciones de educación superior es el manejo de la equidad como promotora de la calidad educativa, derivado de ello se realizan análisis para diagnosticar las políticas acciones y necesidades de las instituciones en este tópico y a partir de esa lógica es que en el Observatorio de Equidad y Calidad en la Educación Superior (ORACLE), nos dimos a la tarea de revisar (de marzo a noviembre 2017) las normativas, documentos y lineamientos que existen en la Universidad Veracruzana para analizar a partir de una reflexión teórica el manejo de este principio en la dinámica institucional.

2. Diagnóstico. Una vez identificado el tópico (equidad y calidad en la Universidad Veracruzana) resolvimos la creación de los instrumentos; en estos identificamos cuatro indicadores: a) pertinencia de las políticas institucionales en el manejo de equidad y calidad en la Universidad Veracruzana, b) la detección de necesidades, c) la opinión de la comunidad académica universitaria acerca de la cultura de equidad, d) la opinión de la comunidad académica universitaria acerca de la cultura de calidad y procedimos a recopilar información y recoger evidencias, que expresen los puntos de vista de los colectivos implicados, bajo el método de diagnóstico participativo propuesto por Nirenberg (2006), realizando un piloteo en marzo 2017 y la aplicación del instrumento en noviembre 2017.

3. Problematización. Para identificar el problema resolvimos que las inconsistencias entre lo que se persigue y lo que en realidad ocurre nos ayudaría a profundizar en el significado de la detección de necesidades; es por ello que decidimos ordenar, agrupar, disponer y relacionar los datos de acuerdo con los objetivos de la investigación, preparando la 
información a fin de proceder a su análisis e interpretación lo que permitió conocer la situación e identificar las problemáticas más recurrentes en la institución.

4. Diseño de una Propuesta de Cambio. En esta fase se consideran las diversas alternativas de actuación y sus posibles consecuencias. Una reflexión prospectiva permite diseñar una propuesta de cambio y mejoramiento, y definir un diseño de evaluación de la misma. Esto se hace con la intención de anticipar los indicadores y metas que darán cuanta del logro de la propuesta y de acuerdo al modelo de investigación revisar la planificación para reorientar la práctica investigativa e interventora.

Población y Muestra. En el campus Poza Rica - Tuxpan, se atiende un total de 7, 642 estudiantes, de los cuales 7,501 pertenecen a 27 programas de nivel licenciatura y 141 corresponden a 10 programas de posgrado; atendidos por 190 Profesores de Tiempo Completo, 48 Técnicos Académicos, 348 de Asignatura, dando un total de 586 académicos en la región.

Para la aplicación de nuestro instrumento realizamos un piloteo en marzo 2017 con un pre diagnóstico a: 14 coordinadores enlace, 8 alumnos consejeros, 5 directores de facultad, 5 secretarios de facultad.

El tipo de muestra utilizada para la investigación fue una muestra no probabilística, que de acuerdo con Hernández, Fernández y Baptista (2014) "selecciona casos o unidades por uno o varios propósitos. No pretende que los casos sean estadísticamente representativos de la población" (p.171). Se utilizó una muestra por conveniencia, por ser una técnica de muestreo no probabilístico y no aleatorio, tomando en cuenta la facilidad de acceso y la disponibilidad de las personas que podían formar parte de la misma, la aplicación del instrumento para el diagnóstico se llevó a cabo en noviembre 2017 a 15 facultades de la región Poza Rica - Tuxpan aplicándose a una población de: 1900 estudiantes, 175 docentes, 15 directores de facultad, 30 elementos del personal administrativo y técnico manual.

\section{Resultados}

La información que se presenta a continuación corresponde a los datos obtenidos del diagnóstico aplicado a los diferentes colectivos que conforman la comunidad académica de la universidad. 
Tabla 1.

Importancia de las políticas de equidad

\begin{tabular}{|c|c|c|c|c|c|c|c|c|c|c|}
\hline $\begin{array}{l}\text { ¿Qué nivel de importancia } \\
\text { tiene para usted que se } \\
\text { promuevan políticas de } \\
\text { equidad en los programas } \\
\text { institucionales de la } \\
\text { Universidad } \\
\text { Veracruzana? }\end{array}$ & 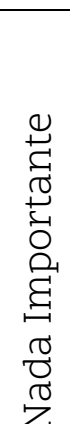 & $\%$ & 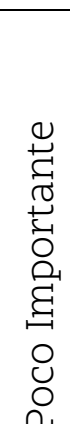 & $\%$ & 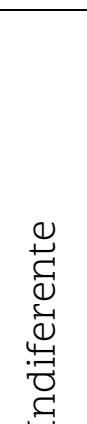 & $\%$ & 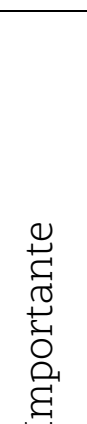 & $\%$ & 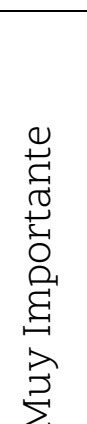 & $\%$ \\
\hline $\begin{array}{l}\text { Personal administrativo } \\
\text { (35 personas) }\end{array}$ & 0 & 0 & 1 & 2.9 & 0 & 0 & 15 & $\begin{array}{l}42 . \\
9\end{array}$ & 19 & $\begin{array}{l}54 . \\
2\end{array}$ \\
\hline $\begin{array}{l}\text { Docentes } \\
\text { (128 docentes) }\end{array}$ & 0 & 0 & 3 & 2.3 & 1 & 0.8 & 37 & $\begin{array}{l}28 . \\
9\end{array}$ & 87 & 68 \\
\hline $\begin{array}{l}\text { Alumnos } \\
\text { (1841 Alumnos) }\end{array}$ & 21 & 1.1 & 29 & 1.6 & 108 & 5.9 & 937 & $\begin{array}{l}50 . \\
9\end{array}$ & 746 & $\begin{array}{l}40 . \\
5\end{array}$ \\
\hline $\begin{array}{l}\text { Funcionarios } \\
\text { (13 funcionarios) }\end{array}$ & 1 & 7.7 & 0 & 0 & 0 & 0 & 2 & $\begin{array}{l}15 . \\
4\end{array}$ & 10 & $\begin{array}{l}76 . \\
9\end{array}$ \\
\hline
\end{tabular}

Fuente: Elaboración propia

Comentario: En relación al nivel de importancia que se otorga a la promoción de políticas de equidad al interior de la institución educativa, la mayoría de las respuestas se concentra en "Importante" y "Muy Importante", lo cual es favorable que la comunidad universitaria le otorgue la importancia que merece.

Sin embargo, hay personas que optaron por las tres respuestas desfavorables ("Nada importante", "Poco importante" e "Indiferente"), en el caso de los alumnos_ 1.1.\% + 1.6\% + 5.9\% $=8.6 \%$ (equivalente a 158 casos) resulta sorprendente el alto número de estudiantes que minimiza la relevancia de promover políticas de equidad, hay que tener presente que ante una desigualdad, este colectivo, es el más vulnerable. En el caso de Docentes _3.1\%, y funcionarios_ $7.7 \%$ es pertinente comentar, que a través de estos se da el modelaje que influye en la actitud de los alumnos, por ello es inconcebible que existan funcionarios o docentes que no concedan importancia a la promoción de políticas de equidad. Por último, en cuanto al personal administrativo (personas que realizan funciones de servicio y que por lo mismo debieran reconocer que el trato igualitario es indispensable en el desempeño de sus actividades), el $2.9 \%$ de este grupo le concede poca importancia a la promoción de políticas de equidad. 
Los datos anteriormente expuestos indican que hacen falta acciones de sensibilización para que el cien por ciento de la comunidad considere que es importante la promoción de políticas de equidad al interior de los programas educativos.

Tabla 2.

Ámbitos de aplicación de la equidad

\begin{tabular}{|c|c|c|c|c|c|c|}
\hline $\begin{array}{l}\text { ¿Cree que la equidad debe aplicarse } \\
\text { solo a cuestiones de género? }\end{array}$ & $\begin{array}{l}\sigma \\
\tilde{D} \\
\tilde{\sigma} \\
z \\
\sigma \\
\tilde{\sigma} \\
\tilde{\nu}\end{array}$ & $\%$ & 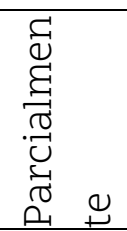 & $\%$ & 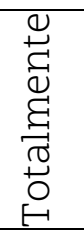 & $\%$ \\
\hline $\begin{array}{l}\text { Personal administrativo ( } 35) \\
(2=5.7 \% \text { personas no contestaron) }\end{array}$ & 24 & 68.6 & 3 & 8.6 & 6 & 17.1 \\
\hline $\begin{array}{l}\text { Docentes }(128) \\
\text { ( } 7 \text { docentes }=5.5 . \% \text { no contestaron) }\end{array}$ & 98 & 76.6 & 14 & 10.9 & 9 & 7 \\
\hline $\begin{array}{l}\text { Alumnos }(1841) \\
(39 \text { alumnos = } 2.1 \% \text { no } \\
\text { contestaron) }\end{array}$ & 1296 & 70.4 & 319 & 17.3 & 187 & 10.2 \\
\hline $\begin{array}{l}\text { Funcionarios } \\
\text { (13 funcionarios) }\end{array}$ & 11 & 84.6 & 1 & 7.7 & 1 & 7.7 \\
\hline
\end{tabular}

Fuente: Elaboración propia.

Comentario: Los datos, en esta interrogante, nos permiten identificar la percepción de los ámbitos de aplicación de la equidad, de los diferentes colectivos encuestados. Si partimos del hecho de que el termino de equidad hace referencia a dar a cada quien lo que necesita, entonces podemos comprender que la equidad no se relaciona exclusivamente con cuestiones de género. Como puede observarse en las cifras, hay integrantes de cada uno de los estamentos que piensan que "Parcialmente" o "Totalmente" la equidad debe aplicarse a solo cuestiones de género, lo que refleja una percepción acotada de las implicaciones de la equidad; si sumamos ambas respuestas tenemos Personal administrativo_25.7\%, Docentes_17.9\%, Alumnos_27.5\% y funcionarios_7.7\%.

Además, es importante comentar el hecho de que hubo docentes, administrativos y estudiantes que no contestaron esta pregunta, lo cual puede conducirnos a dos interpretaciones: una, no tenían el conocimiento para responder o simple y sencillamente fueron indiferentes a este cuestionamiento. 
Tabla 3.

Aplicación de la equidad

\begin{tabular}{|c|c|c|c|c|c|c|}
\hline $\begin{array}{l}\text { ¿Cree que la Universidad } \\
\text { Veracruzana aplica la equidad para } \\
\text { todos los integrantes de la } \\
\text { comunidad universitaria? }\end{array}$ & 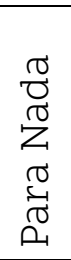 & $\%$ & 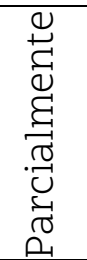 & $\%$ & 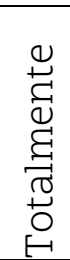 & $\%$ \\
\hline $\begin{array}{l}\text { Personal administrativo (35) } \\
\text { (1 =2.8\%persona no contestó) }\end{array}$ & 5 & 14.3 & 15 & 42.9 & 14 & 40 \\
\hline $\begin{array}{l}\text { Docentes (128) } \\
\text { ( } 3=2.3 \% \text { docentes no contestaron) }\end{array}$ & 6 & 4.7 & 69 & 53.9 & 50 & 39.1 \\
\hline $\begin{array}{l}\text { Alumnos (1841) } \\
\text { (7 = 0.4\% Alumnos no contestaron) }\end{array}$ & 87 & 4.7 & 876 & 47.6 & 871 & 47.3 \\
\hline $\begin{array}{l}\text { Funcionarios } \\
\text { (13 funcionarios) }\end{array}$ & 1 & 7.7 & 4 & 30.8 & 8 & 61.5 \\
\hline
\end{tabular}

Fuente: Elaboración propia.

Comentario: Este cuestionamiento permite apreciar la percepción de los diferentes colectivos, en relación a la aplicación de la equidad, al interior de la comunidad universitaria; el ideal de respuesta en esta interrogante es que todos contestaran "Totalmente"; sin embargo, ninguno de los estamentos analizados contabilizó el 100\% en esta alternativa de respuesta, es más, solo los funcionarios rebasaron el 50\% pero se quedaron en un 61.5\%.

Con estas cifras se puede advertir que gran parte de la comunidad universitaria considera que al interior de la Universidad Veracruzana "Para Nada" o "Parcialmente" se aplica la equidad para todos los integrantes e incluso hubo personas que optaron por no contestar esta pregunta. Se requiere indagar más en los motivos que los conduce a opinar de esta manera. 
Tabla 4.

Aplicación de la equidad en los ámbitos de competencia

\begin{tabular}{|c|c|c|c|c|c|c|}
\hline $\begin{array}{l}\text { ¿Aplica usted equidad en sus } \\
\text { ámbitos de competencia? }\end{array}$ & $\begin{array}{l}\sigma \\
\tilde{0} \\
\tilde{\pi} \\
\tilde{\sigma} \\
\tilde{\sigma} \\
\sigma \\
\tilde{\nu}\end{array}$ & $\%$ & 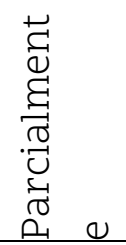 & $\%$ & 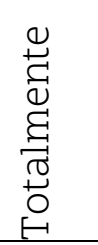 & $\%$ \\
\hline $\begin{array}{l}\text { Personal administrativo ( } 35) \\
\text { ( } 3=8.6 \% \text { personas no contestaron) }\end{array}$ & 2 & 5.7 & 6 & 17.1 & 24 & 68.6 \\
\hline $\begin{array}{l}\text { Docentes (128) } \\
(4=3.1 \% \text { docentes no contestaron) }\end{array}$ & 1 & 0.8 & 19 & 14.8 & 104 & 81.3 \\
\hline $\begin{array}{l}\text { Alumnos (1841) } \\
(18=0.9 \% \text { Alumnos })\end{array}$ & 42 & 2.3 & 701 & 38.1 & 1080 & 58.7 \\
\hline $\begin{array}{l}\text { Funcionarios } \\
\text { (13 funcionarios) }\end{array}$ & 0 & 0 & 1 & 7.7 & 12 & 92.3 \\
\hline
\end{tabular}

Fuente: Elaboración propia.

Comentario: Sin duda alguna la equidad debe estar presente en todas las áreas sustantivas de la Universidad Veracruzana y cobijar a todos los colectivos que la integran. El comportamiento de los datos en ésta pregunta refleja que hay integrantes, de cada uno de los estamentos, que aceptan NO aplicar equidad en su ámbito de competencia o la aplican "Parcialmente", si sumamos los porcentajes de ambas respuestas tenemos que en el caso de los administrativos un 22.8\% más 8.6\% que no contestó es igual a 31.4\%, Docentes 15.6\% más 3.1\% que no contestó suman 18.7\%, Alumnos 40.4\% más 0.9\% que no contestó es igual a 41.3\% y Funcionarios 7.7\%. Las cifras anteriores deberían ser motivo para generar acciones para que el 100\% de los integrantes de la comunidad universitaria aplique la equidad en sus ámbitos de competencia. 
Tabla 5.

Políticas pertinentes en el manejo de la equidad

\begin{tabular}{|c|c|c|c|c|c|c|}
\hline $\begin{array}{l}\text { ¿Considera que la Universidad tiene } \\
\text { políticas institucionales pertinentes } \\
\text { en el manejo de la equidad? }\end{array}$ & 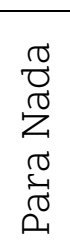 & $\%$ & 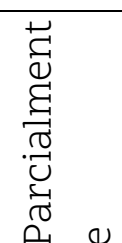 & $\%$ & 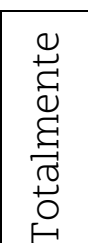 & $\%$ \\
\hline $\begin{array}{l}\text { Personal administrativo ( } 35) \\
\text { ( } 5=14.3 \% \text { personas no contestaron) }\end{array}$ & 2 & 5.7 & 14 & 40 & 14 & 40 \\
\hline $\begin{array}{l}\text { Docentes (128) } \\
\text { ( } 3=2.3 \% \text { docentes no contestaron) }\end{array}$ & 7 & 5.5 & 59 & 46.1 & 59 & 46.1 \\
\hline $\begin{array}{l}\text { Alumnos (1841) } \\
(10=0.5 \% \text { alumnos no contestaron) }\end{array}$ & 94 & 5.1 & 1085 & 58.9 & 652 & 35.5 \\
\hline $\begin{array}{l}\text { Funcionarios } \\
\text { (13 funcionarios) }\end{array}$ & 0 & 0 & 7 & 53.8 & 6 & 46.2 \\
\hline
\end{tabular}

Fuente: Elaboración propia.

Comentario: Como puede observarse la percepción de la comunidad universitaria, especialmente de los alumnos, en relación a las políticas institucionales que promueven la equidad, no es favorable pues no alcanza, en ninguno de los estamentos, ni el 50\%; más bien los porcentajes mayoritarios se inclinan hacia las respuestas de PARA NADA y PARCIALMENTE.

Lo anterior conduce a dos posibles escenarios, uno que hace falta mayor difusión de las políticas de equidad, porque gran porcentaje de la comunidad universitaria está expresando que no son pertinentes las políticas de equidad y dos, que efectivamente hay carencia de políticas institucionales pertinentes en el manejo de la equidad. 
Tabla 6.

Socialización de las políticas institucionales de equidad

\begin{tabular}{|c|c|c|c|c|c|c|}
\hline $\begin{array}{l}\text { ¿Cree que la Universidad socializa } \\
\text { pertinentemente sus políticas } \\
\text { institucionales de equidad y } \\
\text { calidad? }\end{array}$ & 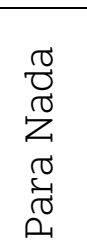 & $\%$ & 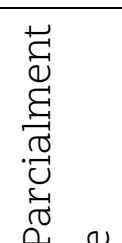 & $\%$ & 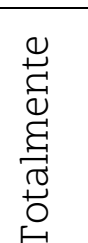 & $\%$ \\
\hline $\begin{array}{l}\text { Personal administrativo ( } 35) \\
\text { ( } 3=8.6 \% \text { personas no contestaron) }\end{array}$ & 1 & 2.9 & 18 & 51.4 & 13 & 37.1 \\
\hline $\begin{array}{l}\text { Docentes ( } 128) \\
\text { ( } 3=2.3 \% \text { docentes no contestaron) }\end{array}$ & 11 & 8.6 & 70 & 54.7 & 44 & 34.4 \\
\hline $\begin{array}{l}\text { Alumnos (1841) } \\
(12=0.6 \% \text { Alumnos no contestaron) }\end{array}$ & 105 & 5.7 & 1135 & 61.7 & 589 & 32 \\
\hline $\begin{array}{l}\text { Funcionarios (13) } \\
\text { (1= 7.7\% funcionarios no contestó) }\end{array}$ & 0 & 0 & 7 & $\begin{array}{l}53.8 \\
\%\end{array}$ & 5 & 38.5 \\
\hline
\end{tabular}

Fuente: Elaboración propia.

Comentario: Las respuestas de esta pregunta, definitivamente reflejan un área débil de la institución, pues en promedio el $35.5 \%$ de la comunidad universitaria, contestó que la institución socializa pertinentemente sus políticas de equidad y calidad. Por otro lado, el promedio de las respuestas desfavorables, es decir, aquellas que marcaron que "Para Nada" o "Parcialmente las socializa" suma el 64.5\% (integrado por el promedio de los que contestaron Parcialmente $55.4 \%$, más $4.3 \%$ promedio de los que consideran Para Nada y más $4.8 \%$ promedio de los que no contestaron).

Los datos de esta interrogante permiten identificar primero que en todos los colectivos hay integrantes que consideran que parcialmente se socializan pertinentemente las políticas institucionales de equidad y calidad y segundo que el porcentaje promedio, de datos desfavorables, es alto $64.5 \%$. 
Tabla 7.

Conocimiento de la política de equidad

\begin{tabular}{|c|c|c|c|c|c|c|}
\hline $\begin{array}{l}\text { ¿Conoce la política institucional en } \\
\text { materia de equidad? }\end{array}$ & $\begin{array}{l}\sigma \\
\sigma \\
\sigma \\
Z \\
\sigma \\
\tilde{\sigma} \\
\sigma_{1}\end{array}$ & $\%$ & 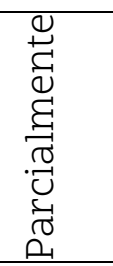 & $\%$ & 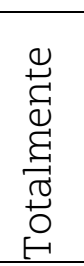 & $\%$ \\
\hline $\begin{array}{l}\text { Personal administrativo (35) } \\
(1=2.9 \% \text { personas) }\end{array}$ & 7 & 20 & 16 & 45.7 & 11 & 31.4 \\
\hline $\begin{array}{l}\text { Docentes (128) } \\
(7=5.5 \% \text { docentes no contestaron) }\end{array}$ & 23 & 18 & 73 & 57 & 25 & 19.5 \\
\hline $\begin{array}{l}\text { Alumnos }(1841) \\
(22=1.2 \% \text { alumnos })\end{array}$ & 566 & 30.7 & 1033 & 56.1 & 220 & 12 \\
\hline $\begin{array}{l}\text { Funcionarios } \\
\text { (13 funcionarios) }\end{array}$ & 1 & 7.7 & 7 & 53.8 & 5 & 38.5 \\
\hline
\end{tabular}

Fuente: Elaboración propia.

Comentario: Los resultados de este cuestionamiento, refleja la correspondencia con las respuestas de la pregunta anterior, pues definitivamente si la comunidad universitaria percibe que no hay socialización de las políticas institucionales, que tienen relación con la equidad entonces, es lógico que no la conozcan, como se puede observar en el grupo más débil (los alumnos) sólo el 12\% respondió que Totalmente la conoce y el 56.1\% la conoce Parcialmente.

Además, el resto de los grupos también merecen atención ya que es inconcebible que los Docentes el 18\% no la conozcan o 57\% la conocen Parcialmente y un 5.5\% optó por no contestar por desconocimiento o apatía (total un 80.5\%). Los administrativos por su parte un $20 \%$ no la conoce, el 45.7\% la conoce Parcialmente y un 2.9\% no contestó (total 68.6\%) y más grave aún, es el hecho de que las personas que representan la autoridad al interior de las entidades educativas expresen: un 7.7\% no la conocen, el 53.8\% la conocen Parcialmente (total 61.5\%). 
Tabla 8. Participación en los programas de promoción de equidad

\begin{tabular}{|c|c|c|c|c|c|c|}
\hline $\begin{array}{l}\text { ¿Le gustaría participar en } \\
\text { programas para promover la } \\
\text { equidad en su dependencia? }\end{array}$ & 䗆 & $\%$ & 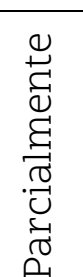 & $\%$ & 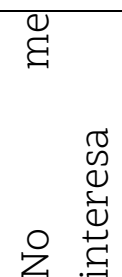 & $\%$ \\
\hline $\begin{array}{l}\text { Personal administrativo (35) } \\
(1=2.9 \% \text { persona no contestó) }\end{array}$ & 15 & 42.9 & 13 & 37.1 & 6 & 17.1 \\
\hline $\begin{array}{l}\text { Docentes }(128) \\
(3=2.3 \% \text { docentes })\end{array}$ & 54 & 42.2 & 50 & 39.1 & 21 & 16.4 \\
\hline $\begin{array}{l}\text { Alumnos (1841) } \\
\text { (31 =1.7\% alumnos) }\end{array}$ & 575 & 31.2 & 766 & 41.6 & 469 & 25.5 \\
\hline $\begin{array}{l}\text { Funcionarios (13) } \\
\text { (1=7.7\% funcionarios) }\end{array}$ & 7 & 53.8 & 4 & 30.8 & 1 & 7.7 \\
\hline
\end{tabular}

Fuente: Elaboración propia.

Comentario: Las respuestas que merecen atención, son las que se concentran en las columnas de No me interesa y Parcialmente, ya que son las que reflejan actitudes desfavorables para participar en programas que promuevan la equidad en su entidad educativa.

Se puede observar que no hay un grupo que quede exento de presencia de integrantes que no le interesa participar, incluso los funcionarios; pero, además, no son porcentajes insignificantes, si sumamos ambas respuestas nos podemos dar cuenta que superan el 50\% entre que no les interesa y parcialmente les interesa.

Un dato útil es el hecho de que es alto el porcentaje de personas que desconocen las políticas institucionales relacionadas con la equidad, como se puede apreciar en las respuestas de la pregunta anterior, pero también es considerable la apatía por participar en programas para promover la equidad en su dependencia, como puede observarse en las respuestas de la presente interrogante.

\section{Reflexiones finales}

La información obtenida en la presente encuesta permite respaldar los siguientes argumentos: 
- En relación al nivel de importancia que cada colectivo otorga a la promoción de políticas de equidad al interior de la institución educativa, puede observarse que un alto número de estudiantes no le conceden relevancia, pero también llama la atención el hecho de que

hay docentes e incluso funcionarios que tampoco le dan importancia, y no hay que perder de vista que el docente es modelo a seguir por parte de los estudiantes y el funcionario, si no le otorga importancia cómo fomentará las políticas institucionales y cómo vigilará que no se transgreda la integridad de los miembros de la comunidad universitaria.

- El que un porcentaje de la comunidad universitaria desconozca las políticas institucionales que tienen relación con la equidad, provoca que se encuentren en una posición de vulnerabilidad ya que no identifican aquellas situaciones que implican una falta de equidad; además, dicho desconocimiento provoca también que existan personas que no apliquen o promuevan la equidad al interior de la institución educativa; y por último, el desconocimiento de las políticas de equidad provoca una visión acotada, relacionando la equidad, únicamente, con cuestiones de género.

- Si bien es cierto que, porcentualmente, los datos duros parecieran indicar que las respuestas obtenidas en el colectivo de los funcionarios son estadísticamente poco representativas es importante hacer mención que la población de funcionarios encuestada fue del total de personas que aplican a este cargo.

- El panorama que se muestra en el presente escrito, demanda realizar acciones de socialización y sensibilización de las políticas institucionales que promueven la equidad.

\section{Referencias}

Hernández R., Fernández C. y Baptista P. (2014) Metodología de la Investigación. 6ạ. Edición. México. Editorial: Mc Graw Hill.

Kemmis, S. McTaggart, R. (1992) Cómo planificar la investigación- Acción. Barcelona. Editorial Laertes.

Latorre, A. (2005) La investigación-acción Conocer y cambiar la práctica educativa. España. 3ra Ed. Editorial Graó.

Nirenberg, O. (2006). El diagnóstico participativo local en intervenciones sociales. Ceadel, Cuaderno No. 44.

Rodríguez, Ruiz Celia (2019) Educación inclusiva. Recomendaciones de la UNESCO para una inclusión educativa. Recuperado de: https://educayaprende.com/educacion-inclusiva/. 
Universidad Veracruzana. (2010). Centro de Desarrollo Humano e Integral de los Universitarios. (2018) Programa Universitario de Educación Inclusiva. Recuperado desde:

https://www.uv.mx/cendhiu/general/quienes-somos-2/

Universidad Veracruzana. (1996) Ley de Autonomía. Recuperado desde: https://www.uv.mx/cq/files/2013/01/Ley-de-Autonomia.pdf

Universidad Veracruzana (2016) Código de ética. Recuperado desde: https://www.uv.mx/legislacion/files/2016/12/Codigo-de-Etica-UV.pdf

Universidad Veracruzana (2016) Programa universitario por una universidad incluyente e integradora de personas con discapacidad. Recuperado de: https://www.uv.mx/rsu/files/2019/01/POR-UNA-UNIVESIDAD-INCLUSIVA-Y ACCESIBLE.-Region-Veracruz.pdf

Universidad Veracruzana (2017) Ley Orgánica. Reimpresión. Recuperado desde: https://www.uv.mx/legislacion/files/2019/04/Ley-Organica-UniversidadVeracruzana-reimpresion2017.pdf

Universidad Veracruzana (2018) Plan Estratégico de Trabajo 2017-2021: Pertenencia y Pertinencia. Recuperado desde: https://www.uv.mx/documentos/files/2019/05/pte2017-2021.pdf

Universidad Veracruzana (2019) Sistema Universitario de Gestión Integral del Riesgo SUGIR. Recuperado desde: https://www.uv.mx/sugir/

Universidad Veracruzana (2019). Centro de Desarrollo Humano e Integral de los Universitarios (CENDHIU). Recuperado de: https://www.uv.mx/cendhiu/cendhiu/atribuciones/ 